\title{
Family Care Map: Sustaining family-centered care in Polytrauma Rehabilitation Centers
}

\author{
James H. Ford II, PhD; ${ }^{1 *}$ Meg Wise, PhD; ${ }^{2}$ Dean Krahn, MD; ${ }^{3-4}$ Karen Anderson Oliver, PhD; ${ }^{3}$ Carmen Hall, \\ RN, PhD; ${ }^{5}$ Nina Sayer, PhD, $\mathbf{L P}^{\mathbf{6}}$ \\ ${ }^{1}$ Center for Health Systems Research and Analysis, and ${ }^{2}$ Sonderegger Research Center, University of Wisconsin-Madi- \\ son, Madison, WI; ${ }^{3}$ William S. Middleton Memorial Veterans Hospital, Madison, WI; ${ }^{4}$ Department of Psychiatry, Univer- \\ sity of Wisconsin-Madison, Madison, WI; ${ }^{5}$ Metropolitan State University, St. Paul, MN; ${ }^{6}$ Center for Chronic Disease \\ Outcomes Research and Polytrauma and Blast-Related Injuries Quality Enhancement Research Initiative, Minneapolis \\ Department of Veterans Affairs Medical Center, Minneapolis, MN
}

\begin{abstract}
The study assessed sustainability of the Family Care Map, a family-centered approach to providing care for Veterans with polytrauma-related injuries, in four Department of Veterans Affairs Polytrauma Rehabilitation Centers. We applied a mixedmethods approach. Staff surveys used standardized measures of sustainability, commitment to change, information, and participation during implementation. Qualitative inquiry assessed Family Care Map implementation and facilitators and barriers to sustainability. Staff sustainability perceptions had a significant positive correlation with affective commitment to change, participation, and information received about the change process. Family Care Map integration into standard practices and use of its concepts with patients and families related to staff perceptions about sustainability. The degree of use and integration of the Family Care Map in traumatic brain injury/polytrauma care varied among the Polytrauma Rehabilitation Centers. Some successful sustainability strategies included integration into daily workflow and organizational culture. Examples of sustainability barriers included staff awareness and use and outdated information. Some practices, such as measuring and documenting the use of the Family Care Map in treatment plans, may not routinely occur. The focus on family-centered care will require further evaluation of organization-, staff-, and innovation-level attributes that influence sustainability of changes designed to improve family-centered care.
\end{abstract}

Key words: affective commitment to change, barriers, Family Care Map, family-centered care, information, participation, polytrauma, program implementation, strategies, sustainability, traumatic brain injury, Veterans.

\section{INTRODUCTION}

Recent wars in Iraq and Afghanistan have exposed Active Duty personnel to blasts from bombs or improvised explosive devices that have resulted in multiple injuries, referred to as "polytrauma." The U.S. Department of Veterans Affairs (VA) defines polytrauma as "two or more injuries to physical regions or organ systems, one of which may be life threatening, resulting in physical, cognitive, psychological, or psychosocial impairments and functional disability" (http://www.queri.research.va.gov/ptbri/). Among Veterans with polytrauma, traumatic brain injury (TBI) is particularly common [1]. In recent reports, 6 to 10 percent of Iraq and

Abbreviations: BNHS-SI $=$ British National Health ServiceSustainability Index, FCM = Family Care Map, IRB = Institutional Review Board, PRC = Polytrauma Rehabilitation Center, PT/BRI = Polytrauma and Blast-Related Injuries, QUERI = Quality Enhancement and Research Initiative, TBI = traumatic brain injury, UW-Madison = University of Wisconsin-Madison, VA = Department of Veterans Affairs.

*Address all correspondence to James $\mathrm{H}$. Ford II, PhD; Center for Health Systems Research and Analysis, University of Wisconsin-Madison, 610 Walnut St, Madison, WI 53726; 608-262-4748.

Email: jhfordii@wisc.edu

http://dx.doi.org/10.1682/JRRD.2014.03.0066 
Afghanistan Veterans who used the VA had a TBI diagnosis in their medical records [1-2], with the majority of these TBIs thought to be mild in severity. Patients with more severe TBI that occurs with polytrauma have complex rehabilitation needs.

Individuals with TBI often have a comorbid diagnosis, such as posttraumatic stress disorder, and need assistance in managing pain [2-7]. The cost per discharge is also approximately three times greater $(\$ 12,000$ vs $\$ 3,900$ per discharge, respectively) for a Veteran with TBI and associated comorbidities than for a Veteran without TBI [2]. To integrate treatment across the continuum of care for Veterans returning from the Iraq and Afghanistan wars with TBI/polytrauma, the VA established the TBI/polytrauma system of care [8-10]. It includes four Polytrauma Rehabilitation Centers (PRCs), one each located in Minneapolis, Minnesota; Palo Alto, California; Richmond, Virginia; and Tampa, Florida, to provide inpatient rehabilitation for Veterans with moderate to severe TBI and polytrauma. A fifth PRC was recently added in San Antonio, Texas. Other components of this system of care include (1) 21 polytrauma rehabilitation network sites to manage the postacute outpatient needs of polytrauma patients, (2) local polytrauma support clinical teams at these outpatient sites, (3) a polytrauma point of contact at all other VA facilities, and (4) polytrauma transitional rehabilitation programs located in each of the PRCs.

The multiple severe injuries that some military personnel incur often place significant burdens on family members (e.g., spouses or parents) to support and provide long-term care to their loved one. The task can be overwhelming [11]. VA staff recognized the importance of including family members in the process of care for individuals with TBI/polytrauma [12]. However, when the war injured were initially coming to the VA for rehabilitation, the approach to family involvement in care varied across the PRCs. Standardization is essential; study results about perceived family needs indicated the importance of developing strategies to reduce stress and address family caregiver requirements associated with providing care for polytrauma patients [12].

To address the variation in how families were integrated into the care processes for Veterans with TBI/polytrauma, the VA implemented a Family Care Quality Improvement collaborative [13]. The collaborative brought together experts from across the VA to establish a standardized protocol for family education and collaboration. It also provided a uniform approach to family-centered care for Veterans with TBI/polytrauma who received inpatient care through a PRC. The result was the development and implementation in 2007 of the Family Care Map (FCM) [10,13]. The six-step FCM outlines a familycentered approach to providing care for Veterans with polytrauma-related injuries. The steps include (1) making first contacts and introductions, (2) welcoming and settling into the PRC, (3) developing a treatment plan, (4) working toward rehabilitation goals, (5) moving forward, and (6) staying connected. The FCM describes transition events between each step and provides detailed information, including "looking ahead" advice for family members. (Please visit http://www.polytrauma.va.gov/FCM/ to learn more about the FCM.) The initial implementation of the FCM has already been described in another research article [13]. However, information on the sustainability of the FCM since its initial implementation is lacking. This information is important because the factors that affect implementation may not be the same as those that affect sustainability over time [14-18]. Healthcare organizations need information on sustainability of patient and familycentered programs to determine how best to allocate resources on an ongoing basis.

Recently, sustainability frameworks have been developed to guide organizational efforts to implement and sustain practices and processes to improve care [15-18]. The frameworks focus on three components of sustainability: organizational capacity to sustain change, attributes of the innovation being implemented, and environmental factors that influence sustainability. Our research focuses on assessing the organizational capacity to sustain the FCM. Information about sustainability might help the VA identify ways to improve processes that strengthen family-centered care, as well as other patient-centered initiatives, for this patient population.

\section{METHODS}

We used a mixed-methods approach involving a Web-based survey followed by key informant interviews in person, by telephone, or in writing. All data were collected between July and August 2012. Researchers from the University of Wisconsin-Madison (UW-Madison) functioned as outside evaluators. The staff surveys did not collect any identifiable information. No identifiable data or information was shared with any VA employees or officials. 


\section{Recruitment Procedures}

VA research staff provided a list of staff members $(N=$ 219) who worked with the PRCs in Minneapolis, Palo Alto, Richmond, and Tampa. Email invitations asked staff to voluntarily complete an anonymous 37-item online survey. Follow-up emails were sent as reminders. For the qualitative inquiry, PRC leadership identified 28 staff to serve as key informants on the FCM within their team.

\section{Survey Measures}

The British National Health Service-Sustainability Index (BNHS-SI) was used to measure the propensity that PRCs will sustain use of the FCM [19-20]. This 10item survey is designed to measure an organization's propensity to sustain an implemented change (Figure). Information about the BNHS-SI has been described in more detail elsewhere [21-22]. Within an organization, a total sustainability propensity score of $<55$ suggests a need to develop an action plan to increase the likelihood that change will be sustained [20]. Within the VA, the BNHS-SI has also been used to assess the likelihood that changes implemented as part of the Mental Health Systems Redesign initiative, as well as changes designed to better integrate primary care and mental health services, would be sustained [22-23].

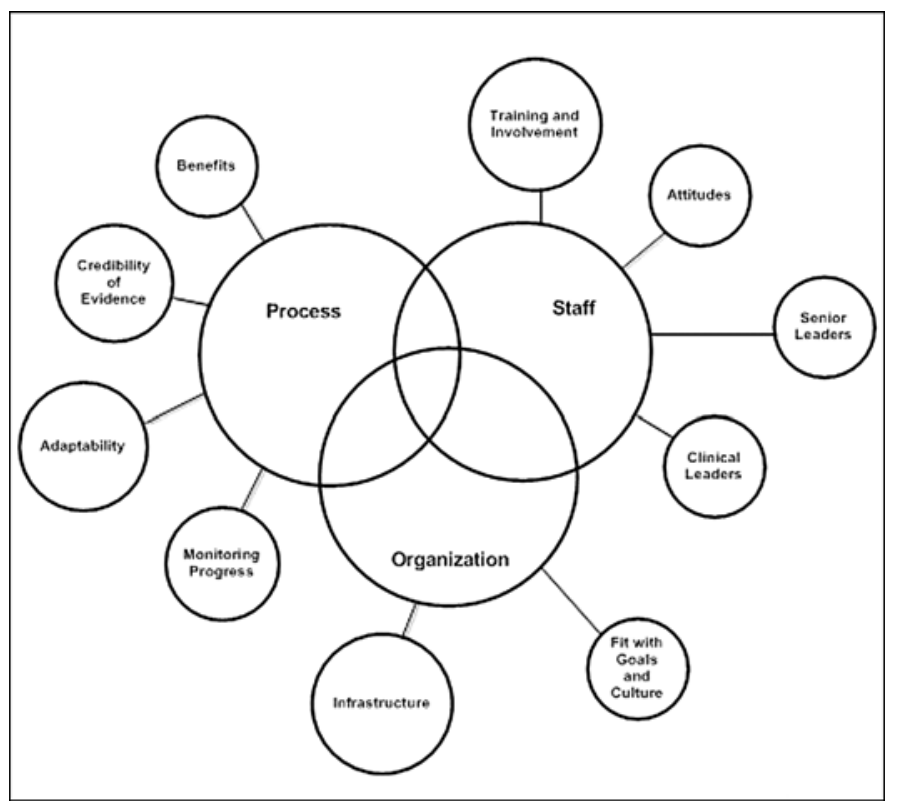

Figure.

British National Health Service-Sustainability Index.
The Web-based survey also measured staff commitment to use of the FCM. To assess commitment, we utilized Herscovitch and Meyer's six-item Affective Commitment to Change scale $(\alpha=0.92)$ [24]. Sample items included "I believe in the value of the Family Care Map" and "The Family Care Map serves an important purpose in the PRC." We also assessed staff perceptions about their level of participation in, as well as information received about, the implementation of the FCM. Participation in implementation of the FCM was measured using a four-question scale $(\alpha=0.72)$ initially developed by Wanberg and Banas [25]. An example question included "I was able to participate in how the Family Care Map is used in my PRC.” We utilized Miller's fourpoint scale ( $\alpha=0.87$ ) to assess staff perceptions about the usefulness of the information PRC staff received during implementation [25-26]. An example question included "The information received about the FCM was useful." Employees used a five-point scale ranging from 1 ("strongly disagree") to 5 ("strongly agree") to respond to the questions in each of these three scales.

In the survey, we wanted to assess staff perceptions about the use of the FCM in their PRC. To achieve this goal, we developed two sets of questions for inclusion in the survey. The first set of questions $(n=10)$ quantified the six steps of the FCM into a question phrased as a specific actionable item. For example, the question for developing a treatment plan was "We work with all TBI/ Polytrauma patients and families to develop a treatment plan.” To these 10 questions, we also added specific FCM questions related to use of the Web site, its applicability in an outpatient setting, use of its concepts when working with patients and families, and the level of integration into standard practices. For these questions, respondents were asked to use a five-point Likert scale (from 5 ["strongly agree"] to 1 ["strongly disagree"]) to indicate the degree to which they agreed with statements about the use of FCM practices in their PRC.

Hall et al. identified 16 specific family-centered practices associated with the implementation of the FCM [13]. We combined similar items from the original list of 16 practices to create a second bank of 12 questions that focused on the use of specific FCM practices within the PRC. For example, the 2 items related to the posting of goals in the patient rooms were combined into 1 specific question, "Goals for patients are posted in their rooms." For each of these questions, the respondents indicated whether the practice was used in the PRC ("yes"), the practice was not used 
("no"), or they did not know whether the practice was used (“do not know”).

Demographic questions included sex, discipline (e.g., physician, nurse, Certified Rehabilitation Registered Nurse, therapist, social worker), job function (e.g., administration/management, direct patient and family care, program support), employment status (e.g., fulltime, part-time), and tenure. We also asked respondents how they would improve the FCM.

\section{Qualitative Inquiry}

The interview questions followed the administration of the Web-based survey and were adapted from our prior mixed-methods investigation on the sustainability of a system redesign project for mental health services across the VA [22]. In this study, we used three approaches in our qualitative inquiry. Due to travel proximity, face-toface interviews were conducted with staff at the Minneapolis PRC. Staff members at the Richmond and Palo Alto PRCs were interviewed by telephone. Both the faceto-face and telephone interviews started with questions designed to get to know the participant (his or her staff position, how he or she got involved with the FCM) and then explored the person's perception of the processes, facilitators, and barriers to implementation and sustainability of the FCM. These interviews ended with a question about how he or she was affected by participation in the FCM and whether there had been any "ah-ha" moments. At the request of the Tampa PRC, we designed a qualitative survey and administered it via SurveyMonkey (Palo Alto, California) to eight staff members. The survey consisted of 16 questions. The questions gathered respondent demographics and assessed staff thoughts about FCM implementation, its use within the PRC, and strategies used to sustain the FCM as well as challenges associated with sustainability.

\section{Data Analysis}

\section{Survey Data}

Survey data were entered into SPSS version 20 (IBM Corporation; Armonk, New York). Descriptive statistics were used to characterize the demographics of the sample and the staff survey responses. We explored correlations between scales and among questions related to sustainability and the perceived use of the FCM.

\section{Qualitative Data}

Two people took notes during the in-person and telephone qualitative interviews. As recommended by Strauss [27], we modified the interview questions to streamline the interview and to explore further themes that emerged after the first set of interviews. We used the qualitative content analysis method to identify themes [28]. Two researchers independently coded the early interviews and discussed the few divergences until agreement was reached. The coding scheme that was developed included a priori themes, based on interview questions regarding implementation, use, and sustainability, and new themes that were grounded in the data (e.g., definition of FCM as protocol or catalyst for family-centered care paradigm shift). We individually coded each interview and then grouped themes by PRC.

\section{RESULTS}

\section{Participant Characteristics}

A total of 44 surveys (20.1\%) were received. Demographic questions were answered for all 44 surveys; however, only 35 surveys had responses for the remaining survey questions. For these 35, distribution of the number of responses by PRC was as follows: PRC1 $(n=6)$, PRC2 $(n=10)$, PRC3 $(n=9)$, and PRC4 $(n=10) .{ }^{*} \mathrm{~A}$ total of 28 people participated in the qualitative inquiry. Table 1 shows the demographics of staff members who completed the survey and of those who completed the qualitative inquiry. The average overall sustainability score across all respondents was 67.97 and ranged from a low of 52.26 in PRC2 to a high of 76.47 in PRC4. Details are available upon request.

\section{Relationship of Commitment, Participation, and Information to Sustainability}

Table 2 shows the correlations between staff perceptions about sustainability and their affective commitment toward, ability to participate in, and belief that they received adequate information about the implementation of the FCM. Affective commitment to change assesses staff perceptions about their desire to support the change based on a belief in its inherent benefits [24]. In this

\footnotetext{
* Site names are intentionally excluded from the article because staff participation in the study was voluntary and confidential. Qualitative and quantitative data were only analyzed by staff from UW-Madison.
} 
Table 1.

Staff survey $(n=44)$ and qualitative inquiry $(n=28)$ response rates and respondent demographics.

\begin{tabular}{|c|c|c|}
\hline $\begin{array}{l}\text { Measure } \\
\end{array}$ & Staff Survey & Qualitative Inquiry \\
\hline \multicolumn{3}{|l|}{ Survey Response by PRC, \% (n) } \\
\hline PRC1 & $18.2(8)$ & $25.0(7)$ \\
\hline PRC2 & $22.7(10)$ & $32.1(9)$ \\
\hline PRC3 & $27.3(12)$ & $14.3(4)$ \\
\hline PRC4 & $31.8(14)$ & $28.6(8)$ \\
\hline \multicolumn{3}{|l|}{ Employee Demographics, \% (n) } \\
\hline Female & $81.8(36)$ & $96.4(27)$ \\
\hline Refused/No Response & $2.3(1)$ & - \\
\hline \multicolumn{3}{|l|}{ Discipline Within VA } \\
\hline Therapist & $27.3(12)$ & $21.4(6)$ \\
\hline RN (Staff Nurse, Case Manager) & $25.0(11)$ & $35.7(10)$ \\
\hline Physician (MD, DO, Physiatrist or Psychiatrist) & $4.6(2)$ & $3.6(1)$ \\
\hline Other & $6.8(3)$ & $3.6(1)$ \\
\hline \multicolumn{3}{|l|}{ Job Classification } \\
\hline Administration/Management & $11.4(5)$ & \\
\hline Clinician & $86.4(38)$ & \\
\hline Clinical Administrator/Management & $2.3(1)$ & \\
\hline \multicolumn{3}{|l|}{ Average Tenure (yr), Mean \pm SD } \\
\hline Facility & $6.8 \pm 5.8$ & $7.4 \pm 5.1$ \\
\hline TBI/Polytrauma & $5.8 \pm 4.6$ & \\
\hline VA & $7.1 \pm 5.6$ & \\
\hline
\end{tabular}

Table 2.

Correlations between staff perceptions about sustainability of Family Care Map and affective commitment toward, participation in, and information about implementation of Family Care Map.

\begin{tabular}{|c|c|c|c|}
\hline Item & $\begin{array}{c}\text { Affective Commitment } \\
\text { to Change }\end{array}$ & $\begin{array}{l}\text { Participation in } \\
\text { Change Process }\end{array}$ & $\begin{array}{c}\text { Information about } \\
\text { Change }\end{array}$ \\
\hline \multicolumn{4}{|l|}{ Affective Commitment to Change } \\
\hline Participation in Change Process & 0.316 & & \\
\hline Sustainability: Benefits of Change & $0.606^{*}$ & $0.341^{\dagger}$ & $0.354 \dagger$ \\
\hline Sustainability: Credibility of Benefits & $0.679^{*}$ & $0.457^{*}$ & $0.499^{*}$ \\
\hline Sustainability: Staff Involvement & 0.211 & $0.716^{*}$ & $0.587^{*}$ \\
\hline Sustainability: Staff Attitudes & $0.472^{*}$ & $0.515^{*}$ & $0.411^{\dagger}$ \\
\hline Sustainability: Senior Leadership & 0.191 & 0.320 & 0.105 \\
\hline Sustainability: Clinical Leadership & 0.048 & $0.364^{\dagger}$ & 0.226 \\
\hline Sustainability: Fit Within Organization & $0.515^{*}$ & $0.427^{\dagger}$ & $0.382^{\dagger}$ \\
\hline
\end{tabular}


study, it is correlated with staff perceptions that the implementation of the FCM would improve efficiency (benefits), that its benefits are clearly apparent and supported by evidence (credibility), and that the implementation of the FCM fits with organizational strategic goals (fit). Affective commitment to change is also related to staff perceptions that they were empowered during the implementation of the FCM and that the implementation process could be adopted to other organizational changes.

Participation in the change process was related to staff involvement and staff attitudes indicating that staff was involved in the implementation of the FCM and felt empowered by that participation. Their participation helped staff understand the benefits of the FCM (credibility) and see that resources were made available and procedures modified as needed to support implementation of the FCM (infrastructure). Finally, participation in the implementation of the FCM was also related to adaptability, which may indicate that the staff saw the value in how its implementation in their PRC could be applied to other organizational changes.

Information received about the implementation of the FCM was related to three questions in the BNHS-SI: credibility, staff involvement, and infrastructure. In this case, information received during staff training about the FCM may have strengthened their perceptions related to staff involvement and enhanced their understanding of the benefits associated with implementing the FCM. Infrastructure changes, such as posting information about the FCM on the unit or in the patient room or incorporating information into the patient's chart process, might have contributed to staff perceptions that they received adequate information about the FCM.

\section{Use of Family Care Map}

Staff responses indicated a high level of agreement (mean $>4.30$ out of 5 ) for five of the six survey questions related to the use of the FCM steps across the four PRCs. The average response to the question "TBI/Polytrauma patients and families often lose contact with their VA Care Team upon discharge from the PRC" was 2.2 out of 5 points, indicating that staff disagreed with this statement. Average staff response to the questions related to the use of the FCM Web site, its applicability in an outpatient setting, use of FCM concepts when working with patients and families, and the level of integration into standard practice ranged from 3.5 to 3.9 points, indicating a moderate level of agreement. Thus, on average, staff agreed that the PRC is currently using 9 of these 10 FCM practices. Details are available upon request.

Table 3 shows the correlation between the use of these 10 practices and the individual BNHS-SI questions. Interestingly, the use of the FCM when working with patients and their families (item $\mathrm{J}$ in Table 3 ) is significantly correlated with 8 of the 10 BNHS-SI questions. The exceptions were senior leadership support and organizational fit (i.e., fit with strategic plans), which may indicate that staff were unsure whether the FCM is a strategic priority and therefore supported by senior leadership. Staff perceptions related to benefits, credibility, staff involvement, and staff attitudes are significantly correlated with staff beliefs that the ideas in the FCM have been integrated into standard practices within the PRC (item H in Table 3).

The use of FCM practices varied within the PRC depending on the specific practice. For example, approximately 9 out of 10 respondents believed that their admission procedures accommodated family preferences and that patients were assigned a staff point of contact at admission. However, approximately one in three respondents said that their PRC was giving families a copy of the FCM, and almost 50 percent did not know or were unsure whether their PRC was documenting and tracking the use of the FCM. Full results are shown in Table 4.

\section{Qualitative Inquiry}

Table 5 shows the findings from the qualitative inquiry. We use four categories to discuss the responses from the qualitative inquiry. In relation to the FCM, these categories are (1) implementation facilitators and barriers, (2) use and benefits, (3) sustainability strategies, and (4) barriers to sustainability.

\section{Implementation Facilitators and Barriers}

Sixteen people (57.1\% of the interviewees) reported that they had been involved with the implementation of the FCM. Frequently mentioned facilitators of implementation included perception that the FCM was a useful tool ( $n=10)$, structured method of pictorially representing it during team meetings $(n=7)$, discussion in team meetings $(n=6)$, clinical leadership support $(n=5)$, and perception that the FCM provided structure to how the team was already doing work $(n=4)$.

The key barrier seemed to be staff time and competing responsibilities. As stated by one respondent, it was "hard to get volunteers because they do not get compensated for their time or it may depend on when the group meets. We 
Table 3.

Correlations between use of Family Care Map (FCM) practices and sustainability items.

\begin{tabular}{|c|c|c|c|c|c|c|c|c|c|c|}
\hline \multirow{2}{*}{ Sustainability Item } & \multicolumn{10}{|c|}{ Use of FCM Item } \\
\hline & $\mathbf{A}$ & B & C & D & $\mathbf{E}$ & $\mathbf{F}$ & G & $\mathbf{H}$ & I & $\mathbf{J}$ \\
\hline Benefits & $0.360^{*}$ & -0.053 & -0.137 & -0.065 & -0.096 & -0.055 & $0.405^{*}$ & $0.469^{\dagger}$ & $-0.389^{*}$ & $0.439^{\dagger}$ \\
\hline redibility & 0.030 & -0.056 & -0.104 & -0.004 & -0.013 & -0.024 & $0.467^{\dagger}$ & $0.557^{\dagger}$ & $-0.421^{*}$ & $0.591^{\dagger}$ \\
\hline Adaptability & -0.194 & -0.151 & -0.136 & -0.089 & -0.204 & 0.130 & 0.211 & $0.336^{*}$ & $-0.415^{*}$ & $0.406^{*}$ \\
\hline ffec & 0.013 & -0.096 & -0.050 & -0.025 & -0.178 & 0.045 & -0.050 & 0.327 & -0.179 & $0.395^{*}$ \\
\hline taff Involvement & 0.039 & 0.123 & 0.184 & 0.088 & 0.062 & -0.214 & 0.088 & $0.477^{\dagger}$ & -0.214 & $0.626^{\dagger}$ \\
\hline taff Attitudes & 0.159 & -0.019 & -0.093 & -0.026 & -0.143 & -0.034 & 0.41 & $0.505^{\dagger}$ & -0.331 & $0.513^{\dagger}$ \\
\hline nio & 0.065 & -0.192 & -0.140 & -0.088 & -0.182 & -0.015 & -0.0 & 0.094 & -0.178 & 0.191 \\
\hline linical Leade & -0.054 & -0.157 & 0.115 & 0.069 & -0.014 & -0.144 & -0.140 & -0.138 & -0.218 & $0.367^{*}$ \\
\hline rganization & 0.119 & -0.048 & -0.120 & -0.115 & -0.169 & 0.012 & $0.384^{*}$ & 0.293 & $-0.547^{\dagger}$ & 0.257 \\
\hline nfrastructure for Change & 0.010 & 0.113 & 0.194 & 0.227 & 0.105 & -0.118 & 0.080 & 0.266 & $-0.348^{*}$ & $0.672^{\dagger}$ \\
\hline
\end{tabular}

Note: Items related to use of FCM are-

A. Prior to admission, we try to contact and introduce all traumatic brain injury (TBI)/polytrauma patients and family to Polytrauma Rehabilitation Center (PRC) Care Team.

B. We have specific plan to welcome and settle all TBI/polytrauma patients and families into PRC.

C. We work with all TBI/polytrauma patients and families to develop treatment plan.

D. We regularly encourage and assist all TBI/polytrauma patients and families in working toward their rehabilitation goals.

E. We routinely communicate with and answer questions from TBI/polytrauma patients and families to assist with transition to next level of care.

F. TBI/polytrauma patients and families often lose contact with their Department of Veterans Affairs Care Team upon discharge from PRC.

G. I think FCM should be adapted for use with outpatients with TBI/polytrauma.

H. Ideas in FCM have been integrated into standard practices within our PRC.

I. I do not use FCM as Web-based tool.

J. My team uses concepts in FCM in our work with patients and families.

${ }^{*}$ Correlation significant at 0.05 level (2-tailed).

${ }^{\dagger}$ Correlation significant at 0.01 level (2-tailed).

Table 4.

Routine Polytrauma Rehabilitation Center use of Family Care Map (FCM) practices $(n=35)$.

\begin{tabular}{|c|c|c|c|}
\hline \multirow{2}{*}{ Practice } & \multicolumn{3}{|c|}{ Staff Response, \% (n) } \\
\hline & Yes & No & Do Not Know \\
\hline 1. Family members are informed about FCM Web site and how to access it. & $54.3(19)$ & $11.4(4)$ & $34.3(12)$ \\
\hline 2. Families receive hard copy of FCM. & $37.1(13)$ & $17.1(6)$ & $45.7(16)$ \\
\hline 3. New staff orientation introduces FCM. & $45.7(16)$ & $25.7(9)$ & $28.6(10)$ \\
\hline 4. Our admission procedures accommodate family preferences. ${ }^{*}$ & $91.2(31)$ & $5.9(2)$ & $2.9(1)$ \\
\hline 5. All patients are assigned staff point of contact at admission. & $91.4(32)$ & $5.7(2)$ & $2.9(1)$ \\
\hline 7. Our medical record uses designated note to document staff and family interactions. & $54.3(19)$ & $34.3(12)$ & $11.4(4)$ \\
\hline 8. We have incorporated FCM into weekly interdisciplinary care plan meetings. & $62.9(22)$ & $22.9(8)$ & $14.3(5)$ \\
\hline 9. We document and track use of FCM. & $34.3(12)$ & $37.1(13)$ & $28.6(10)$ \\
\hline 10. FCM stage is recorded in interdisciplinary treatment plan. & $51.4(18)$ & $25.7(9)$ & $22.9(8)$ \\
\hline 11. Family goals for patients are incorporated into patient's treatment plan. & $82.9(29)$ & $17.1(6)$ & $0.0(0)$ \\
\hline
\end{tabular}

have been really busy lately and no one wants to come to the meeting." Other barriers included challenges in accessing information about the FCM through its Web site, staff turnover, and the family being overwhelmed by the FCM.
We discovered some variance within the PRCs about the use and perceived value of the FCM. These differences seemed to be based on the informants' position and tenure within the PRC. In general, social workers/case 
JRRD, Volume 51, Number 8, 2014

Table 5.

Qualitative inquiry results: Family Care Map (FCM) implementation and sustainability.

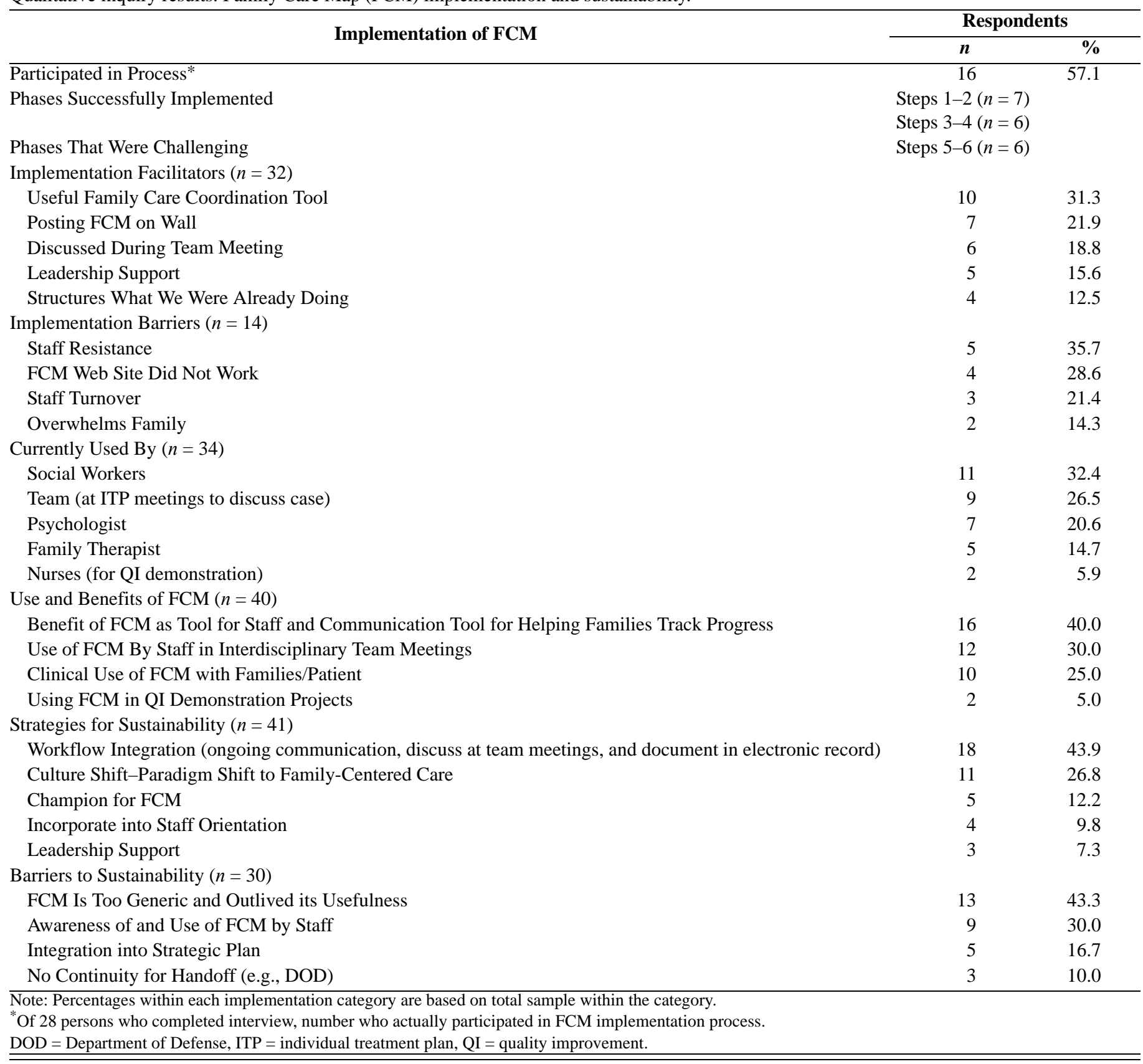

managers, psychologists, and family therapists used the FCM to communicate with families and to guide discussions in interdisciplinary team meetings. After implementation was finished, staff identified that the steps from first contact to rehabilitation (steps 1 through 4) were those running smoothly in the PRC. For example, one respondent said, "Transitions from other hospitals is going 100 times more smoothly with families than before." Another respondent stated, "I would say phases 1-4 are generally smooth for the most part. There are exceptions, but the majority of patients and families are eager to engage in rehab and to improve." When asked about which steps in the FCM were challenging to implement, 6 out of the 13 staff responding indicated that the later steps focused on discharge and postdischarge were the most difficult. One respondent said, "Families are 
afraid to leave the facility no matter how much education is given. Some families are ready and pushing to leave, but this is the minority."

\section{Use and Benefits}

The staff identified four attributes related to the use and benefits of the FCM that may also support its sustained use in the PRCs. The most frequently mentioned is that the staff saw the benefit of the FCM as a tool for helping families and patients $(n=16)$. A specific way that the FCM helps families is as a communication tool. Using the FCM, staff can help families understand where their loved one is along the continuum of care during their rehabilitation process. For example, one respondent indicated, "We have become simpler and more concrete with family. Goals are posted in [the patient] room. We communicate verbally and visually with patients and families." Other identified ways that the FCM was used included in interdisciplinary team meetings $(n=12)$, clinically with families and patients $(n=10)$, and in quality improvement demonstration projects $(n=2)$.

\section{Sustainability Strategies}

We discovered general agreement that the FCM had been sustained in each PRC. Multiple strategies were associated with its sustainability.

The most frequently mentioned strategy for sustaining use of the FCM was workflow integration $(n=18)$. Processes included ongoing team communication through team meetings and by documenting patient progress in the electronic health record. Discussions about patient progress across the FCM steps, such as the completion of treatment goals (step 4) and readiness to transition to outpatient care (step 5) were important in workflow integration. For example, one respondent stated, "I can facilitate or give ideas about how to do it (sustain the Family Care Map) but I imagine that it is charting that will help with sustainability." Another PRC had integrated the FCM into weekly interdisciplinary team meetings where assigning a stage elicited considerable discussion, planning, and dialog. Informants from this PRC noted that "The Family Care Map is common sense stuff — it was not new-but it gave it a measureable way where you can point to an actual level" or "Family centered care has always been there, but the FCM . . . maintains communication among the team.” The different steps in the FCM also help the team focus on patient progress. For example, one respondent said, "It was a great idea to have the components separated out to explore at the individual client level. As it was played out in the Interdisciplinary Planning meetings, we could discuss at the level of each [of the FCM] phases in terms of how we approach treatment."

Institutionalization of a change into the culture of an organization is another key strategy for sustainability. Respondents across the PRCs indicated that the ongoing use of the FCM created a culture shift in the organization toward family-centered care $(n=11)$. For example, one respondent indicated that "If we removed the FCM, the processes would keep going. [Family centered care] has always been there, but the FCM has given it structureweekly evaluation-what goals, plans for given stage.” Other interviews suggested that even though new staff might not recognize the FCM model, care to patients and families clearly functioned with a family-centered mission and incorporated FCM themes into service delivery. These thoughts were echoed in the sentiments of another respondent who said, "Since its inception it has been discussed. It is something that we use and its benefits are there for the patient and family. It helps to keep the professional mind focused on the patient's progress." Respondents from another PRC explored questions about the role and definition of the FCM as the focus of a change. People in this organization described FCM as a "catalyst for shifting the paradigm of polytrauma care from patient centered to family centered." Other staff stated that the "FCM was an early family-centered care tool-the single rose has blossomed to two dozen roses." Yet another respondent from the PRC noted that she knew the FCM was a success because "We no longer need it. Family-centered care is now the way things are done."

In addition to these strategies, respondents indicated that organizational attributes often associated with successful implementation also affected sustainability. These included (1) the presence of a champion, (2) incorporation into new staff orientation, and (3) leadership support. A champion plays an important role in ensuring that a tool continues to be utilized. Since social workers were identified as the staff that currently use the FCM, it is not surprising that they were seen as its champions. A respondent from one PRC summed it up in saying, "Social workers are most aware of the phases - coordinate the next steps." One informant noted that the nurses with longer tenure on her unit found the family-focus foreign, but new graduates had a philosophy of family-centered care-thus, staff turnover was a facilitator rather than a barrier to change. 
Leadership can also help support sustainability by taking responsibility for the efforts to sustain the change process in such a way that staff will generally share information with and actively seek advice from leadership. For example, a respondent from one PRC stated, "The new director is very supportive, shows up at the interdisciplinary team meeting, and makes decisions that support the whole team by working to meet the needs of the team, patient, and family.”

\section{Barriers to Sustainability}

The most common barrier to sustainability of the FCM was that some staff believed that it needed to be updated and further developed to maintain relevance ( $n=$ 13). That is, staff may now have integrated the core concepts underlying the original FCM and need a more sophisticated clinical tool. In particular, the usefulness of the FCM for those patients who return to Active Duty after their stay in the PRC was questioned; PRC staff may have difficulty staying connected with these patients (step 6 of the FCM). It was also unclear whether use of the FCM had been integrated into the strategic plan or goals of the organization.

\section{DISCUSSION}

Results from the qualitative interviews indicate that the FCM was seen more as a tool to facilitate a paradigm shift rather than as a change in healthcare delivery procedures. The FCM reinforced or moved the PRC toward family-centered care and, as a result, created a shift in the organizational culture. Efforts to sustain changes requiring coordination across multiple stakeholders within an organization are influenced by the match between organizational culture and the change (i.e., the FCM) being implemented [16-17,29]. While integration into the culture is an important attribute of sustainability, it is less clear from the survey results whether specific practices (e.g., documenting staff and family interactions) are being applied consistently across the PRCs.

As a tool, the FCM is too simplistic, because the family-centered principles of the care map have been institutionalized. While the care teams are more familycentered, only some practices associated with the FCM are being widely implemented. For example, respondents indicated that each PRC routinely assigns a staff point of contact, incorporates family goals for the patient into their treatment plan, and posts goals in the patient's room. These findings suggest that these practices have become standard practice - an important aspect of sustainability [29-30]. However, several practices (see items 7-10 in Table 4) related to the documentation or measurement of the FCM have not been widely adapted. For each practice, only 34 to 54 percent of the staff indicated that these practices were being done in their PRC. Research indicates that ongoing measurement and feedback or issues associated with measuring the effect of change serve as both facilitators and barriers to sustainability of change in a quality improvement collaborative [16,31-36]. The absence of such documentation or measurement could be an indicator that practices may not be sustainable over the long run, especially if new staff do not receive an orientation to the FCM.

The overall sustainability propensity ( $\mu=67.97)$ of the FCM is higher than previous VA sustainability studies related to mental health and system redesign projects $(\mu=61.36)$ and primary care and mental health integration projects $(\mu=55.50)$ within the VA [21-22]. It may be in part because the FCM was seen as a tool or catalyst for adapting practices in each PRC that are consistent with the organizational culture. Across the three VA studies, we may be seeing cultural versus individual differences associated with the sustainability of a specific change. The qualitative findings lend some support to this concept, because we believe that some sites have fully implemented the FCM and its practice, but the staff may not recognize the FCM as the instrument associated with this new and institutionalized family-oriented care. Further exploration of how the change influences culture and then sustainability should be explored.

Research suggests that staff training and involvement during implementation facilitates sustainability [3032,36]. Our results indicate that staff perceptions of involvement in and information received about the FCM during implementation are significantly associated with their perceptions about sustainability. For example, staff perceptions about active participation may have enhanced their understanding of and belief in how the FCM would improve efficiency and make their jobs easier when working with Veterans and their families. Active participation is also an intrinsic staff motivator that increases the likelihood that a change will be sustained [19,37]. Interview results reinforce this concept because staff indicated the perceived usefulness of the FCM as a tool not only for staff but also for communication with family members 
about patient progress. These beliefs were grounded in their active involvement during the implementation process and supported by a champion for sustainability, most often the social worker, and through an active integration into staff workflow during team meetings and documentation in the clinical notes. Despite these findings, more prospective research is needed to fully understand how staff engagement during implementation influences longterm sustainability and how it may differ for a family-centered versus a specific process (e.g., reducing wait times) change.

\section{LIMITATIONS}

The survey response rate is limited. While we can infer about differences across PRCs, the small sample size from each PRC limits the ability to test for significant differences. For example, the total sustainability score for PRC2 is 15 to 24 points lower than for the other three PRCs, suggesting that the staff have a different opinion about the likelihood that the FCM might be sustained. The low response rate most likely represents opinions of staff (i.e., champions, members of the collaborative) who were most intimately involved in the FCM implementation process. As a result, the small sample size in each PRC may not reflect the opinions of all staff working in each PRC as it relates to the sustainability of the FCM. The process for conducting the qualitative interviews is also another limitation. Logistical considerations allowed for in-person interviews to be conducted at PRC2. However, we were limited to telephone interviews at two other PRCs and an online survey at the final PRC. Research has found that responses are similar for information collected by telephone or in person [38-39], except for more personal information [40]. However, it is unclear whether online survey responses to the same questions yield similar results.

\section{CONCLUSIONS}

The Affordable Care Act authorized funding to support patient-centered outcomes research (www.pcori.org). A primary focus is on the inclusion of key stakeholders (e.g., physicians, patients, and families) in the provision of care. The FCM is just one example of how families are integrated into the care of Veterans. This research provides insights into efforts to sustain the use of the FCM. It highlights staff perceptions about the use of family-centered practice, as well as their suggestions for improving the FCM. However, it is also important to recognize that tools such as this one require constant monitoring and updates if they are to continue to be useful and sophisticated clinical tools. These efforts may include incorporating these family-centered principles across the entire continuum of care, inpatient as well as outpatient, for individuals with TBI. Outside the VA, other organizations may be implementing new family-centered care. Findings from this research provide insights on how to effectively implement and sustain this approach to care. Specifically, it implies that organizations that are introducing practices related to familycentered care should develop an implementation strategy. This strategy should ensure that new staff are oriented to and trained in how to document the use of family-centered care activities. It should also make information about family-centered care more readily available to staff and family members; involve staff, especially nurses, during implementation; and identify a champion for family-centered care (including leadership support) for the change. This implementation strategy should also integrate the concepts and principles of family-centered care into not only the daily workflow but also the culture of the organization. As more attention is paid to patient centeredness of care, it will become increasingly important for researchers to study and evaluate the attributes at the organizational, staff, and innovation levels that influence the sustainability of changes designed to improve patient- and familycentered care.

\section{ACKNOWLEDGMENTS}

\section{Author Contributions:}

Study concept and design: J. H. Ford II, C. Hall, D. Krahn, K. A. Oliver, N. Sayer.

Acquisition of data: J. H. Ford II, K. A. Oliver, M. Wise. Analysis and interpretation of data: J. H. Ford II, K. A. Oliver, M. Wise.

Drafting of manuscript: J. H. Ford II, M. Wise.

Critical revision of manuscript for important intellectual content: J. H. Ford II, C. Hall, D. Krahn, K. A. Oliver, N. Sayer, M. Wise.

Study supervision: J. H. Ford II, K. A. Oliver.

Financial Disclosure: The authors have declared that no competing interests exist.

Funding/Support: This material was based on work supported by the VA Polytrauma and Blast-Related Injuries (PT/BRI) Quality Enhancement and Research Initiative (QUERI) Locally Initiated Project (grant 
QLP 56-002). Dr. Ford's work was also supported in part by the National Institute of Drug Abuse (grant 5 R01 DA020832).

Additional Contributions: The authors would like to thank Maureen Fitzgerald for her assistance in the review of this article. The authors also thank the staff at the Minneapolis, Palo Alto, Richmond, and Tampa PRCs for the generous support of this project in answering our questions about the sustainability of the FCM. When the study was conducted, Dr. Hall was the Implementation Research Coordinator for the VA's PT/BRI QUERI. Dr. Krahn is now Acting Director, Office of Mental Health Operations, VA Central Office, and Dr. Ford is now with the Center for Health Systems Research and Analysis, UWMadison.

Institutional Review: The UW-Madison Health Sciences and Minneapolis VA Medical Center Institutional Review Boards (IRBs) classified this project as Quality Improvement and therefore exempted it from IRB review.

Participant Follow-Up: The authors do not plan to inform participants of the publication of this study because contact information is unavailable.

Disclaimer: The views expressed in this article are those of the authors and do not necessarily reflect the position or policy of the VA or U.S. Government, and no official endorsement should be inferred.

\section{REFERENCES}

1. Cifu DX, Taylor BC, Carne WF, Bidelspach D, Sayer NA, Scholten J, Campbell EH. Traumatic brain injury, posttraumatic stress disorder, and pain diagnoses in OIF/OEF/OND Veterans. J Rehabil Res Dev. 2013;50(9):1169-76. [PMID:24458958] http://dx.doi.org/10.1682/JRRD.2013.01.0006

2. Taylor BC, Hagel EM, Cutting A, Carlson KF, Cifu DX, Bidelspach DE, Sayer NA. Fiscal year 2011 VA utilization report for Iraq and Afghanistan War veterans diagnosed with TBI. Prepared for the VA Polytrauma and BlastRelated Injuries QUERI \#PLY 05-2010-2 [Internet]. Washington (DC): Department of Veterans Affairs; 2012 Sep. Available from: http://www.queri.research.va.gov/ptbri/ docs/FY11-TBI-Diagnosis-HCU-Report.pdf

3. Clark ME, Bair MJ, Buckenmaier CC 3rd, Gironda RJ, Walker RL. Pain and combat injuries in soldiers returning from Operations Enduring Freedom and Iraqi Freedom: Implications for research and practice. J Rehabil Res Dev. 2007;44(2):179-94. [PMID:17551872] http://dx.doi.org/10.1682/JRRD.2006.05.0057

4. Dobscha SK, Campbell R, Morasco BJ, Freeman M, Helfand MV. Evidence-based synthesis program reports. Pain in patients with polytrauma: A systematic review. Portland (OR): Portland Department of Veterans Affairs Healthcare System, Oregon Evidence-based Practice Center; 2008.

5. Institute of Medicine. Returning home from Iraq and Afghanistan: Assessment of readjustment needs of veter- ans, service members, and their families. Washington (DC): The National Academies Press; 2013.

6. Lew HL, Poole JH, Vanderploeg RD, Goodrich GL, Dekelboum S, Guillory SB, Sigford B, Cifu DX. Program development and defining characteristics of returning military in a VA Polytrauma Network Site. J Rehabil Res Dev. 2007;44(7):1027-34. [PMID:18075959] http://dx.doi.org/10.1682/JRRD.2007.05.0073

7. Lew HL, Otis JD, Tun C, Kerns RD, Clark ME, Cifu DX. Prevalence of chronic pain, posttraumatic stress disorder, and persistent postconcussive symptoms in OIF/OEF veterans: Polytrauma clinical triad. J Rehabil Res Dev. 2009; 46(6):697-702. [PMID:20104399] http://dx.doi.org/10.1682/JRRD.2009.01.0006

8. Benedict SM, Scholten J. The Veterans Health Administration's Polytrauma System of Care: Rehabilitation for today's and tomorrow's veterans. Generations. 2010; 34(2):106-8.

9. Belanger HG, Uomoto JM, Vanderploeg RD. The Veterans Health Administration's (VHA's) Polytrauma System of Care for mild traumatic brain injury: Costs, benefits, and controversies. J Head Trauma Rehabil. 2009;24(1):4-13. [PMID:19158591] http://dx.doi.org/10.1097/HTR.0b013e3181957032

10. Griffin JM, Friedemann-Sánchez G, Hall C, Phelan S, van Ryn M. Families of patients with polytrauma: Understanding the evidence and charting a new research agenda. J Rehabil Res Dev. 2009;46(6):879-92.

[PMID:20104409] http://dx.doi.org/10.1682/JRRD.2008.08.0104

11. Sayer NA, Cifu DX, McNamee S, Chiros CE, Sigford BJ, Scott S, Lew HL. Rehabilitation needs of combat-injured service members admitted to the VA Polytrauma Rehabilitation Centers: The role of PM\&R in the care of wounded warriors. PM R. 2009;1(1):23-28. [PMID:19627869] http://dx.doi.org/10.1016/j.pmrj.2008.10.003

12. Friedemann-Sánchez G, Sayer NA, Pickett T. Provider perspectives on rehabilitation of patients with polytrauma. Arch Phys Med Rehabil. 2008;89(1):171-78. [PMID:18164350] http://dx.doi.org/10.1016/j.apmr.2007.10.017

13. Hall C, Sigford B, Sayer N. Practice changes associated with the Department of Veterans Affairs' Family Care Collaborative. J Gen Intern Med. 2010;25(Suppl 1):18-26. [PMID:20077147] http://dx.doi.org/10.1007/s11606-009-1125-3

14. Damschroder LJ, Aron DC, Keith RE, Kirsh SR, Alexander JA, Lowery JC. Fostering implementation of health services research findings into practice: A consolidated framework for advancing implementation science. Implement Sci. 2009;4:50. [PMID:19664226] http://dx.doi.org/10.1186/1748-5908-4-50 
15. Aarons GA, Hurlburt M, Horwitz SM. Advancing a conceptual model of evidence-based practice implementation in public service sectors. Adm Policy Ment Health. 2011; 38(1):4-23. [PMID:21197565] http://dx.doi.org/10.1007/s10488-010-0327-7

16. Chambers DA, Glasgow RE, Stange KC. The dynamic sustainability framework: Addressing the paradox of sustainment amid ongoing change. Implement Sci. 2013; 8:117. [PMID:24088228] http://dx.doi.org/10.1186/1748-5908-8-117

17. Schell SF, Luke DA, Schooley MW, Elliott MB, Herbers $\mathrm{SH}$, Mueller NB, Bunger AC. Public health program capacity for sustainability: A new framework. Implement Sci. 2013;8:15. [PMID:23375082] http://dx.doi.org/10.1186/1748-5908-8-15

18. Scheirer MA, Dearing JW. An agenda for research on the sustainability of public health programs. Am J Public Health. 2011;101(11):2059-67. [PMID:21940916] http://dx.doi.org/10.2105/AJPH.2011.300193

19. Davies B, Edwards N. The action cycle: Sustain knowledge use. In: Strauss S, Tetroe J, Graham ID, editors. Knowledge translation in health care: Moving from evidence to practice. Oxford (UK): Wiley-Blackwell; 2009. p. 165-73.

20. Maher L, Gustafson D, Evans A. Sustainability. Leicester (UK): British National Health Service Modernisation Agency; 2004.

21. Doyle C, Howe C, Woodcock T, Myron R, Phekoo K, McNicholas C, Saffer J, Bell D. Making change last: Applying the NHS institute for innovation and improvement sustainability model to healthcare improvement. Implement Sci. 2013;8(1):127. [PMID:24160758] http://dx.doi.org/10.1186/1748-5908-8-127

22. Ford JH 2nd, Krahn D, Wise M, Oliver KA. Measuring sustainability within the Veterans Administration Mental Health System Redesign initiative. Qual Manag Health Care. 2011;20(4):263-79. [PMID:21971024] http://dx.doi.org/10.1097/QMH.0b013e3182314b20

23. Ford JH 2nd, Krahn D, Oliver KA, Kirchner J. Sustainability in primary care and mental health integration projects in Veterans Health Administration. Qual Manag Health Care. 2012;21(4):240-51. [PMID:23011071] http://dx.doi.org/10.1097/QMH.0b013e31826d1d1a

24. Herscovitch L, Meyer JP. Commitment to organizational change: Extension of a three-component model. J Appl Psychol. 2002;87(3):474-87. [PMID:12090605] http://dx.doi.org/10.1037/0021-9010.87.3.474

25. Wanberg CR, Banas JT. Predictors and outcomes of openness to changes in a reorganizing workplace. J Appl Psychol. 2000;85(1):132-42. [PMID:10740964] http://dx.doi.org/10.1037/0021-9010.85.1.132

26. Miller VD, Johnson JR, Grau J. Antecedents to willingness to participate in a planned organizational change. J Appl
Commun Res. 1994;22:59-80.

http://dx.doi.org/10.1080/00909889409365387

27. Strauss AL. Qualitative analysis for social scientists. Cambridge (UK): Cambridge University Press; 1987.

28. Hsieh HF, Shannon SE. Three approaches to qualitative content analysis. Qual Health Res. 2005;15(9):1277-88. [PMID:16204405] http://dx.doi.org/10.1177/1049732305276687

29. Scheirer MA. Linking sustainability research to intervention types. Am J Public Health. 2013;103(4):e73-80. [PMID:23409904] http://dx.doi.org/10.2105/AJPH.2012.300976

30. Parand A, Benn J, Burnett S, Pinto A, Vincent C. Strategies for sustaining a quality improvement collaborative and its patient safety gains. Int J Qual Health Care. 2012; 24(4):380-90. [PMID:22669328] http://dx.doi.org/10.1093/intqhc/mzs030

31. Parand A, Dopson S, Vincent C. The role of chief executive officers in a quality improvement initiative: A qualitative study. BMJ Open. 2013;3(1):e001731 [PMID:23293245] http://dx.doi.org/10.1136/bmjopen-2012-001731

32. Bray P, Cummings DM, Wolf M, Massing MW, Reaves J. After the collaborative is over: What sustains quality improvement initiatives in primary care practices? JT Comm J Qual Patient Saf. 2009;35(10):502-8. [PMID:19886089]

33. Carlhed R, Bellman C, Bojestig M, Bojö L, Peterson A, Lindahl B; Quality Improvement in Coronary Care (QUICC) Study Group. Quality improvement in coronary care: Analysis of sustainability and impact on adjacent clinical measures after a Swedish controlled, multicenter quality improvement collaborative. J Am Heart Assoc. 2012;1(4):e000737. [PMID:23130153]

http://dx.doi.org/10.1161/JAHA.112.000737

34. Chin MH, Kirchhoff AC, Schlotthauer AE, Graber JE, Brown SE, Rimington A, Drum ML, Schaefer CT, Heuer LJ, Huang ES, Shook ME, Tang H, Casalino LP. Sustaining quality improvement in community health centers: Perceptions of leaders and staff. J Ambul Care Manage. 2008; 31(4):319-29. [PMID:18806592] http://dx.doi.org/10.1097/01.JAC.0000336551.67922.2f

35. Øvretveit J, Klazinga N. Linking research to practice: The organisation and implementation of The Netherlands health and social care improvement programmes. Health Policy. 2013;109(2):175-86. [PMID:23270882] http://dx.doi.org/10.1016/j.healthpol.2012.11.005

36. Schneider KL, Agins BD, Ng DW, Monserrate JM, Hirschhorn LR. Evaluation of regional HIV provider quality groups to improve care for people living with HIV served in the United States. J Health Care Poor Under- 
JRRD, Volume 51, Number 8, 2014

served. 2012;23(3 Suppl):174-92. [PMID:22864496] http://dx.doi.org/10.1353/hpu.2012.0142

37. Parker LE, de Pillis E, Altschuler A, Rubenstein LV, Meredith LS. Balancing participation and expertise: A comparison of locally and centrally managed health care quality improvement within primary care practices. Qual Health Res. 2007;17(9):1268-79. [PMID:17968043] http://dx.doi.org/10.1177/1049732307307447

38. Bjorner JB, Rose M, Gandek B, Stone AA, Junghaenel DU, Ware JE Jr. Difference in method of administration did not significantly impact item response: An IRT-based analysis from the Patient-Reported Outcomes Measurement Information System (PROMIS) initiative. Qual Life Res. 2014;23(1):217-27. [PMID:23877585] http://dx.doi.org/10.1007/s11136-013-0451-4

39. Fine TH, Contractor AA, Tamburrino M, Elhai JD, Prescott MR, Cohen GH, Shirley E, Chan PK, Goto T, Slembarski R, Liberzon I, Galea S, Calabrese JR. Validation of the telephone-administered PHQ-9 against the in-person administered SCID-I major depression module. J Affect Disord. 2013;150(3):1001-7. [PMID:23747208] http://dx.doi.org/10.1016/j.jad.2013.05.029

40. Ezzati M, Martin H, Skjold S, Vander Hoorn S, Murray CJ. Trends in national and state-level obesity in the USA after correction for self-report bias: Analysis of health surveys. J R Soc Med. 2006;99(5):250-57.

[PMID:16672759]

http://dx.doi.org/10.1258/jrsm.99.5.250

Submitted for publication March 3, 2014. Accepted June 6, 2014.

This article and any supplementary material should be cited as follows:

Ford JH 2nd, Wise M, Krahn D, Oliver KA, Hall C, Sayer N. Family Care Map: Sustaining family-centered care in Polytrauma Rehabilitation Centers. J Rehabil Res Dev. 2014;51(8):1311-24.

http://dx.doi.org/10.1682/JRRD.2014.03.0066

ResearcherID/ORCID: James H. Ford II, PhD: C-74172014

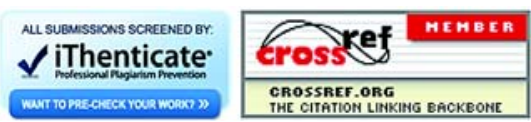

